\title{
Computed tomography scan radiation overdose: a case discussion of recurrent neurocardiogenic syncope underwent to 112 brain computed tomography scans in three years
}

\author{
Behçet Al ${ }^{1, *}$, Suleyman Ercan ${ }^{2}$, Suat Zengin ${ }^{1}$, Vedat Davutoglu ${ }^{2}$, Hasan Kiliç ${ }^{1}$, Remzi Yigiter $^{3}$ \\ ${ }^{1}$ Emergency Department of Medicine School in Gaziantep Üniversitesi, Turkey \\ ${ }^{2}$ Cardiology Department of Medicine School in Gaziantep Üniversitesi, Turkey \\ ${ }^{3}$ Neurology Department of Gaziantep Avukat Cengiz State Hospital, Turkey
}

Email address:

behcetal@gmail.com (Behçet Al)

To cite this article:

Behçet Al, Suleyman Ercan, Suat Zengin, Vedat Davutoglu, Hasan Kiliç, Remzi Yigiter. Computed Tomography Scan Radiation Overdose: a Case Discussion of Recurrent Neurocardiogenic Syncope Underwent to 112 Brain Computed Tomography Scans in Three Years, Science Journal of Public Health. Vol. 1, No. 1, 2013, pp. 47-50. doi:10.11648/j.sjph.20130101.18

\begin{abstract}
A 32-year-old female patient who was diagnosed with mixed type neurocardiogenic syncope and used to have epileptic seizures 4-6 times per day, admitted to emergency rooms with headache, dizziness, syncope, and post-ictal syndromes, and was exposed to high doses of radiation will be discussed in this study together with radiation overdose. Despite her diagnosis was certain, due to inadequate uptake of the medical history and incomplete physical examination, the patient had a total of 112 computed tomography scans within the past 36 months, $80 \%$ of which were performed in emergency services. Our purpose of presenting this case is to draw attention to the fact that treating patients in emergency services without learning full medical history of patients and without completing proper physical examination may prompt more detailed investigation then required. To our knowledge of the literature, there is no other case admitted to the emergency rooms due to syncope and exposed to such high doses of ionizing radiation.
\end{abstract}

Keywords: Radiation Overdose, Syncop, Computed Tomography Scan, Emergency Department

\section{Introduction}

Computed tomography (CT) scanning is an invaluable tool to the medical practitioner and its diagnostic utility has revolutionized current medical practice. However, all too often scans are ordered or repeated without considering the consequence to the patient $(1,2)$. Over the past 2 decades, CT has changed the landscape of diagnostic radiology fundamentally. Technological advancements in multidetector helical imaging permit fast and easy delivery of detailed crosssectional images to the radiologist, often making CT the primary diagnostic tool of choice (3). Applications are more varied than ever, ranging from presurgery diagnosis of appendicitis in children to whole-body imaging of healthy, asymptomatic adults as a preventive measure. Beyond diagnostic applications, CT imaging is used in cancer staging, coronary artery calcium scoring, treatment planning in radiation oncology, fluoroscopy, guidance and placement procedures and composite imaging with positron emission tomography (PET) for 3-D and functional images (4). Although CT is a remarkably reliable tool, as well as user-friendly for both physicians and technologists, it is not without drawbacks. The primary problem with CT is that it exposes patients to high levels of x-ray radiation. X-rays are a form of ionizing radiation, which is known to increase risk of cancer (5).

With an approximate 62 million CT scans preformed in the United States in 2006 (an increase of 50\% from 2000 estimates) and $45 \%$ of the collective radiation dose attributed to CT scanning alone $(6,7)$. NCRP, the Food and Drug Administration, and the National Cancer Institute $(8,9)$ believe that the smallest dose of low-level radiation has the potential to cause a small increase in health risks to humans. It is suggested that the biological effects of ionizing radiation may has as high as 1 fatal cancer for every $1000 \mathrm{CT}$ scans. To keep discussion of potential cancer risks from CT in proper perspective, it is important to recognize that the lifetime risk of cancer is quite high in the general popula- 
tion. As a consequence, prenatal effects (deterministic effect) such as acute radiation syndrome, radiation burns, fibrosis, necrosis, sclerosis, radioactive intoxications, mental retardation and teratogenic malformation and cancers (cytocastic effect) such as leukemia, lung, gastrointestinal and thyroid cancers are the majority encountered complications of ionized radiation (10).

\section{Case}

A thirty-two-year-old female nurse was admitted to our emergency department because of syncope. At the time of admission, the patient's seizure had ended, she was conscious and her Glakow Coma Scale was 15. All vital signs of the patient were stable and laboratory values were within normal limits. Her history was that she had an epileptic seizure and fell five years ago, and hit her head on the ground. She developed post traumatic subarachnoid hemorrhage (SAH). Following that, she developed hydrocephalus and retinal detachment in the left eye. After developing SAH, she started to have seizures 15-18 times a month, and sometimes 7-8 times a day; her seizures lasted about 2-3 minutes and spread to her entire body. The patient was prescribed carbamazepine $400 \mathrm{mg} 2 \times 1$ and lamotrigine $150 \mathrm{~m} \mathrm{2x} 1$. The patient was diagnosed with mixed type neurocardiogenic syncope and was implanted with a Guidant DDD-R pacemaker. In the same year she was given a retinal detachment surgery and a ventriculoperitoneal (VP) shunt was inserted. Her shunt had to be replaced four times in four years due to infection and obstruction. Upon increases in the patient's pain, pethidine HCL was started at a special medical center and pethidine HCL dependence developed; consequently, the patient began psychiatric treatment for that. Two years after the SAH, the patient developed a subdural hematoma and she underwent surgical operation. In the third year of her disease, the patient gave birth to a 32-week healthy baby by cesarean section while she had the pacemaker implant. During pregnancy CT scans were suspended. Currently, the patient has attacks of epileptic seizures 4-6 times a day on average; she less frequently suffers from headache, nausea and vomiting, and gait disturbance. In approximately three years, the patient was admitted to emergency services $(60 \%$ state hospital, $40 \%$ university hospital) in many occasions with headache, dizziness, syncope, and post-ictal syndromes. During this period, despite she had a diagnosed disease; she had a total of 112 computed tomography scans, $80 \%$ of which took place in emergency services, due to inadequate uptake of the medical history and incomplete physical examination. Eight of these CT scans were performed during the assessments of VP shunts, two were for SAH assessment purposes, two were before subdural hemorrhage and in control assessments; and one was performed during the retinal detachment operation. The remaining $99 \mathrm{CTs}$ were performed during epileptic seizures, in the course of admittance to the hospital with headache or dizziness; and no new pathology was detected in any of these scans. Consent of neither the patient nor the patient's relatives was sought in any of the CT scans. When we investigated the root cause of so many computed tomography scans, the three reasons turned up:

1. Incomplete medical history,

2. Inadequate examinations, and most importantly,

3. Related clinics' (neurology and brain surgery) refusal to evaluate the patient without a CT scan.

The patient has been followed oncologically (lungs, gastrointestinal tract, cardiac) and hematologically for the last one year. There has been no pathological finding until now; however, the patient was recommended to have checks every 6 months.

The patient was invited along with her family to our emergency department and was introduced to all emergency service personnel and especially to physicians; the personnel was informed of the status of the patient. It was explained that unnecessary tomography scans should be avoided to prevent the patient's exposure to more radiation. Follow-ups were recommended to be performed from a single center. The patient was warned to carry a note on herself stating "I am a neurocardiogenic syncope patient. Please do not perform CT scan on me without my or my husband's consent." This attempt was successful. The patient did not have CT scans in her admittances to the emergency rooms due to seizures in the last 12 months.

\section{Discussion}

CT constitutes the most comprehensive use of ionizing radiation in today's clinical practices and its use is increasing rapidly. In the last two decades, the use of CT increased 12 times in the UK and 20 times in the USA $(10,11)$. The increased CT use was particularly observed in pediatric patients (from $6 \%$ to $11 \%$ ) (12). When compared with conventional $\mathrm{x}$-ray examinations, patients are exposed to much higher doses of radiation in BT scans. For example, the organ dose in a chest X-ray is approximately $0.01-0.15$ mGy, while it is $10-20 \mathrm{mGy}$ in a thorax CT. As a matter of fact, this dose increases up to approximately $80 \mathrm{mGy}$ in 64 cross-section CT-coronary angiography (13). The amount of organ dose received in CT depends on various factors such as the size of the patient's body, section thickness, the number of slices, the pitch value, the tube voltage, and current values. Ideally, a separate scan protocol should be planned for each individual and for each examination. However, this practice is not possible within the heavy workflow (14). No appropriate protocol setting was determined and implemented during any of the CT scans of our patient. The number of examination is an important factor affecting the amount of the dose received. Mettler et al. in their study determined that $30 \%$ of the cases had three scans, $7 \%$ had five scans, and $4 \%$ had more than nine scans on the same day (12). Unlike the available literature, our patient had an average of $37.3 \mathrm{CT}$ scans per year and an average of 3.1 CT scans per month. Such high frequency of CT scans was not found in the literature. 
All solid cancer risks were found to be increasing with radiation dose in $\mathrm{CT}$ scans, especially in children (10). According to the data obtained, $0.4 \%$ of cancers in the U.S. observed between 1991-1999 were reported to be caused by the use of CT (15). At present, this ratio is considered to be between $1.5 \%$ to $2 \%$. However, the epidemiological studies conducted for this purpose are not yet sufficient (16). Therefore, the studies conducted after the atom bomb explosions are considered as the gold standard for quantitative assessment of cancers induced by low doses of radiation (17). Putative low-dose radiation is between 5-200 $\mathrm{mSv}[(1$ sievert $=1$ gay $(\mathrm{Gy})]$ and this dose is equivalent to the dose received in one or more CT examinations. SmithBindman et al. retrospectively examined over 1,000 CT scans in four different centers and calculated the expected cancer risk according to the radiation doses received. The average effective dose received is calculated to be $2-3 \mathrm{mSv}$ in routine cranial CT scan and $31 \mathrm{mSv}$ in multiphasic abdominal-pelvic CT scan. In addition, the calculated cancer risks differed depending on the type of examination, patient's age and gender. For example, the risk of developing cancer after a coronary CT-angiography performed on a 40year-old female patient was $1 / 270$ and 1/8100 after a cranial CT scan whereas on a male patient of the same age, these probabilities were calculated to be $1 / 600$ and $1 / 11080$, respectively. However, these risks were calculated to be twice as much for a 20 -year-old patient and $50 \%$ lower for a 60 -year-old patient (18). Although CT scans have significantly increased relatively in our country, we were not able to access any statistical data. Accordingly, our patient has been exposed to radiation at a dose of approximately 224$336 \mathrm{mSv}$ (corresponds to the radiation dose of 2,240-3,360 chest X-ray scans on average). Compared with the results of the previous study, our patient's risk of developing cancer was calculated to be $11 / 675$ depending on 112 CT scans performed in the last three years. This is a considerably high probability. Cancer screening and controls on our patient are yet new (one year), she must be followed-up for a very long time. In the study of Berrington de Gonzalez et al., the expected risk of cancer due to CT scans is $0.0004 \%$ (19). In Brenner et al.'s study, the risk of cancer was calculated to be $1 / 550$ in children having abdominal CT and $1 / 1500$ in children having cranial CT scans (20). There are also other studies which report that the risk is $1 / 143$ for a twenty-year-old woman while the risk is $1 / 3261$ for an 80 year-old male patient (21).

Although the CT radiation dose is much higher than other imaging modalities, this fact is ignored. While the organ dose is approximately $3 \mathrm{mGy}$ in two-way bilateral mammography, this dose is approximately $10 \mathrm{mGy}$ in adult abdominal CT whereas it is about $20 \mathrm{mGy}$ in abdominal CT scans of the newborn. In a study by Slovis et al., it was established that $1 / 3$ of CT scans were unnecessary (22). In our case, the rate of unnecessary CT scans is much higher. No new pathology was detected apart from the previous sequels in the 99 CT scans. Physicians who request the highest number of CT scans are not fully aware of the risks.
In a study conducted between radiologists and emergency physicians in the United States, the radiation dose of CT examination had been overlooked in both groups at a rate of $75 \% .53 \%$ of radiologists and $91 \%$ of emergency physicians do not believe the risk of cancer increases with $\mathrm{CT}$ scans (23). In our patient, $90 \mathrm{CT}$ scans were performed upon admittance to emergency services, including $36 \mathrm{CT}$ scans performed in the service where we work. The patient was not directed by the emergency service personnel for oncological or hematological screening on any account. This finding supports the results of the literature that emergency physicians do not believe in an increased risk of cancer based on CT examinations. To prevent further malpractice, a seminar was organized for introduction of the patient and the patient relatives to emergency service personnel. In the syncopal episodes observed within the last one year, emergency personnel demonstrated resistance against the pressure from related consulting departments and did not perform CT scans.

\section{Suggestions for Minimizing Risks}

CT utilizing or tissue dose should be decreased, and the lowest dose should be used to have sufficient image (24). $\mathrm{MR}$, ultrasound and lower-dosage conventional radiography should be fully considered as an alternative to CT. Also, individualized technical protocols that minimize radiation exposure for each examination should be used. Patients should be informed specifically about the dangers of radiation exposure; disclosure forms that explain radiation risks are preferred (24). All CT examinations must be allowed only after a medical consultation (4). Brochures relevant to radiation risks should be published and distributed to the physicians. Last, all health care providers must adhere to the ALARA (as low as reasonably achievable) standard (24).

\section{As a Result}

The ease and speed of a CT examination should not replace a thorough patient history and complete physical examination. Although BT has important diagnostic medical benefits, it constitutes great risk for the formation of radiation-related cancer. Prior to deciding for CT use, the benefit - cost ratio should be considered properly. Good clinical approach and other imaging methods should be used prior to promulgation of indication. If CT scan is absolutely necessary, then automatic dose control methods or appropriate scanning parameters should be selected. It should be common knowledge that children and women are at greater risk and patients are exposed to high doses of radiation in screening programs; so, the necessity of scan should be questioned.

\section{References}


[1] Baskerville JR Screening patients with multi-detector computed axial tomography (MDCT): when will we inform patients about the risk of radiation? Emerg Med J, 2008; 25(6): 323-324.

[2] Heilman RS CT: time to face the facts. Radiographics, 2006: 26(4): 1133.

[3] Hughes J. CT: Radiation overdose? Radiol Technol. 2008; 80(1): 92-4.

[4] Yoshizumi TT, Nelson RC. Radiation issues with multidetector row helical CT. Crit Rev Comput Tomogr. 2003; 44: 95-117.

[5] Brenner D, Hall E. Computed tomography - an increasing source of radiation exposure. N Engl J Med. 2003; 357: $2277-2284$

[6] Lee CI, Haims AH, Monico EP, Brink JA, Forman HP. Diagnostic CT scans: assessment of patient, physician, and radiologist awareness of radiation dose and possible risks. Radiology, 2004; 231(2): 393-398.

[7] Wiest PW, Locken JA, Heintz PH, Mettler FA Jr. CT scanning: a major source of radiation exposure. Semin Ultrasound CT MR, 2002; 23(5): 402-410.

[8] Linton OW, Mettler Jr FA. National conference on dose reduction in $\mathrm{CT}$, with an emphasis on pediatric patients. AJR Am J Roentgenol 2003; 181: 321 - 9.

[9] Rice HE, Frush DP, Farmer D, Waldhausen JH; APSA Education Committee. Review of radiation risks from computed tomography: essentials for the pediatric surgeon. J Pediatr Surg. 2007; 42(4): 603-7.

[10] Hall EJ, Brenner DJ. Cancer risks from diagnostic radiology. Br J Radiol.2008; 81(965): 362-78.

[11] Berrington de Gonzalez A, Mahesh M, Kim KP, Bhargavan M, Lewis R, Mettler F, Land C. Projected cancer risks from computed tomographic scans performed in the United States in 2007. Arch Intern Med. 2009; 169(22): 2071-7.

[12] Mettler FA Jr, Wiest PW, Locken JA, Kelsey CA. CT scanning: patterns of use and dose. J Radiol Prot 2000; 20: 3539.
[13] What's NEXT? Nationwide Evaluation of X-ray Trends: 2000 computed tomography. (CRCPD publication no. NEXT 2000CTT.) Conference of Radiation Control Program Directors, Department of Health and Human Services, 2006. Mettler FA Jr, Wiest PW, Locken JA, Kelsey CA. CT.

[14] Paterson A, Frush DP, Donnelly LF. Helical CT of the body: are settings adjusted for pediatric patients? AJR Am J Roentgenol 2001; 176: 297-301.

[15] Berrington de Gonzalez A, Darby S. Risk of cancer from diagnostic X-rays: estimates for the UK and 14 other countries. Lancet 2004; 363: 345-51.

[16] Giles J. Study warns of 'avoidable' risks of CT scans. Nature 2004; 431: 391.

[17] Işık Z, Selçuk H, Albayram S. Bilgisayarlı Tomografi ve Radyasyon. Klinik Gelişim Dergisi, 2010; 23(2): 16-19.

[18] Smith-Bindman R, Lipson J, Marcus R, Kim KP, Mahesh, Gould R, Berrington de Gonzalez A, Miglioretti DL. Radiation dose associated with common computed tomography examinations and the associated lifetime attributable risk of cancer. Arch Intern Med. 2009; 169(22): 2078-86.

[19] Berrington de Gonzalez A, Mahesh M, Kim KP, Bhargavan M, Lewis R, Mettler F, Land C. Projected cancer risks from computed tomographic scans performed in the United States in 2007. Arch Intern Med. 2009 Dec 14; 169(22): 2071-7.

[20] Brenner DJ, Elliston CD, Hall EJ, Berdon WE. Estimates of the cancer risks from pediatric CT radiation are not merely theoretical. Med Phys 2001; 28: 2387-8.

[21] White KS. Helical/spiral CT scanning: a pediatric radiology perspective. Pediatr Radiol 1996; 26: 5-14.

[22] Slovis TL, Berdon WE. Panel discussion. Pediatr Radiol 2002; 32: 242-4.

[23] Lee CI, Haims AH, Monico EP, Brink JA, Forman HP. Diagnostic $\mathrm{CT}$ scans: assessment of patient, physician, and radiologist awareness of radiation dose and possible risks. Radiology 2004; 231: 393-8.

[24] Brenner DJ, Hall EJ. Computed tomography-an increasing source of radiation exposure. N Engl J Med. 2007 Nov 29; 357(22): 2277- 84. 